\title{
Fully Developed Free Convection Flow in a Vertical Slot with Open to Capped Ends: D'Alembert's Approach
}

\author{
Basant Kumar Jha, Michael Oluwakayode Oni* \\ Department of Mathematics, Ahmadu Bello University, Zaria, Nigeria \\ Email address: \\ basant777@yahoo.co.uk (B. K. Jha), michaeloni29@yahoo.com (M. O. Oni) \\ ${ }^{*}$ Corresponding author
}

To cite this article:

Basant Kumar Jha, Michael Oluwakayode Oni. Fully Developed Free Convection Flow in a Vertical Slot with Open to Capped Ends: D’Alembert's Approach. Mathematical Modelling and Applications. Vol. 2, No. 5, 2017, pp. 52-56. doi: 10.11648/j.mma.20170205.12

Received: September 26, 2017; Accepted: October 27, 2017; Published: November 20, 2017

\begin{abstract}
The fully developed free convection flow in a vertical slot with open to capped ends discussed by Weidman [5] and Magyari [6] is scrutinized in this present work. Exact solution of momentum and energy equations under relevant boundary conditions as discussed in $[5,6]$ is obtained using the D'Alembert's method. Numerical comparison of this present work is made with previous result of [6] and the results were justified using the well-known implicit finite difference method (IFDM); this gives an excellent comparison. During the course of numerical investigation, it is found that D'Alembert's approach is a simpler, reliable and accurate tool for solving coupled equations.
\end{abstract}

Keywords: Fully Developed, Free Convection, Vertical Slot, Capped Ends, D’Alembert’s Approach

\section{Introduction}

Laminar flow between two differentially-heated vertical plates is a classical problem in free convection flow due to its applications in industrial and technological world.

A lot of researchers have worked on fluid flow when both ends are capped as well as when both ends are opened. Batchelor [1] gives a detailed analysis of the conduction and convection regime flow whereas the analytical studies and experimental values of $\tau$ were carried out by Elder [2]. Daniel [3] discussed a transition from the conductive to convective regime for closed cavities for flow with large Prandtl numbers. Bühler [4] reported a significant result of stable laminar convection in a tall slender cavity. For conduction regime flow, he proposed a generalized case by assuming the ends of the channel to be porous.

Weidman [5] presented a convection regime flow in a vertical slot: continuum of solutions from capped to open ends and found that identical viscous and thermal boundary layer exist at the opposing walls when the cavity is capped. In addition, he concluded that as the flow evolves to one with open ends, there is intensification (attenuation) of the boundary layers near the hot (cold) walls. Furthermore, Magyari [6] revisited the work of Weidman [5] and presented another approach "normal modes" of solution to the coupled energy and momentum equations. The conduction regime is seen to correspond exactly with Weidman [5]. Other research articles related to the present student can be found in [7-9]

The aim of this present work is to present an alternative approach (D'Alembert's method) to derive exact solutions for the mathematical model presented in $[5,6]$. The governing momentum and energy equations are solved exactly and the impact of all pertinent parameters are discussed with the aid of line graph.

\section{Mathematical Analysis}

This work is related to the recent works of Bühler [4], Weidman [5] and Magyari [6] in which they investigated the conduction [4] and the convection [5] regime of the free convection flow in a differentially heated tall vertical slot with open to capped ends. For the quasi-static transition from the open to the capped end situation, a continuum of solutions has been obtained for both cases.

This present work makes use of D'Alembert's approach used by Jha [10] to solve the same problem discussed in [5, 6]. Following the model and assumptions presented by 
Weidman and Magyari $[5,6]$, we have

$$
\begin{aligned}
& \frac{d^{2} U}{d Y^{2}}+G \theta=0 \\
& \frac{d^{2} \theta}{d Y^{2}}-E U=0
\end{aligned}
$$

Along with the boundary conditions $[5,6]$

$$
\begin{gathered}
\mathrm{U}(-1 / 2)=0, \theta(-1 / 2)=-\theta_{0} \\
\mathrm{U}(1 / 2)=0, \theta(1 / 2)=1-\theta_{0}
\end{gathered}
$$

where $E$ and $G$ are the Elder and Grashof numbers respectively defined by [5] as

$$
\mathrm{E}=\frac{\beta \nu d}{\kappa \Delta T}=\operatorname{Pr} \tau, G=\frac{g \gamma T d^{3}}{v^{2}} \text { and } \tau=\frac{\beta}{\Delta T / d}=\frac{C}{B} U_{1}=\frac{U}{\sqrt{G / 4 E}}(4)
$$

In order to solve equations (1) and (2), we choose the D'Alembert's method as discussed by Jha and Apere [10]. We multiply equation (1) by $A$ and add it to equation (2)

$$
\frac{\mathrm{d}^{2}[\mathrm{AU}+\theta]}{\mathrm{dY^{2 }}}-[\mathrm{GA} \theta-\mathrm{EU}]=0
$$

Also the boundary conditions (equation 3 ) become

$$
\begin{array}{r}
\operatorname{AU}(-1 / 2)+\theta(-1 / 2)=-\theta_{0} \\
\operatorname{AU}(1 / 2)+\theta(1 / 2)=1-\theta_{0}
\end{array}
$$

where

$$
\mathrm{A}=\frac{-E}{G A}
$$

The general solution of equation (5) with boundary conditions (6) is

$$
\mathrm{AU}+\theta=\frac{\sin (\sqrt{G A}(Y+1 / 2))}{\sin (\sqrt{G A})}-\frac{\theta_{0} \cos (Y \sqrt{G A})}{\cos (\sqrt{G A} / 2)}
$$

From equation (7), we have

$$
\mathrm{A}^{2}=-\frac{E}{G}
$$

The roots of equation (9) are

$$
\mathrm{A}_{1}, A_{2}= \pm i \sqrt{\frac{E}{G}}
$$

Replacing $A$ with $A_{1}$ and $A_{2}$ one after the other in equation (8), we obtained

$$
\begin{aligned}
& \mathrm{A}_{1} \mathrm{U}+\theta=\frac{\sin \left(\delta_{1}(Y+1 / 2)\right)}{\sin \left(\delta_{1}\right)}-\frac{\theta_{0} \cos \left(Y \delta_{1}\right)}{\cos \left(\delta_{1 / 2}\right)} \\
& \mathrm{A}_{2} \mathrm{U}+\theta=\frac{\sin \left(\delta_{2}(Y+1 / 2)\right)}{\sin \left(\delta_{2}\right)}-\frac{\theta_{0} \cos \left(Y \delta_{2}\right)}{\cos \left(\delta_{2} / 2\right)}
\end{aligned}
$$

$$
\delta_{1}=\sqrt{G A_{1}} \text { and } \delta_{2}=\sqrt{G A_{2}}
$$

Solving equations (11) and (12) simultaneously gives the velocity and temperature distributions respectively as:

$$
\begin{gathered}
\mathrm{U}(\mathrm{Y})=\frac{1}{A_{1}-A_{2}}\left[\frac{\sin \left(\delta_{1}(Y+1 / 2)\right)}{\sin \left(\delta_{1}\right)}-\frac{\sin \left(\delta_{2}(Y+1 / 2)\right)}{\sin \left(\delta_{2}\right)}+\theta_{0}\left(\frac{\cos \left(Y \delta_{2}\right)}{\cos \left(\delta_{2} / 2\right)}-\right.\right. \\
\left.\left.\frac{\cos \left(Y \delta_{1}\right)}{\cos \left(\delta_{1} / 2\right)}\right)\right] \\
\theta(Y)=\frac{1}{A_{2}-A_{1}}\left[A_{2} \frac{\sin \left(\delta_{1}(Y+1 / 2)\right)}{\sin \left(\delta_{1}\right)}-A_{1} \frac{\sin \left(\delta_{2}(Y+1 / 2)\right)}{\sin \left(\delta_{2}\right)}+\right. \\
\left.\theta_{0}\left(A_{1} \frac{\cos \left(Y \delta_{2}\right)}{\cos \left(\delta_{2} / 2\right)}-A_{2} \frac{\cos \left(Y \delta_{1}\right)}{\cos \left(\delta_{1} / 2\right)}\right)\right]
\end{gathered}
$$

If $E=0$ from Eq. (10), then $A_{1}=A_{2}=\delta_{1}=\delta_{2}=0$

Then the solution of equation (2) with boundary conditions (3) becomes

$$
\theta(Y)=\left(\frac{1}{2}-\theta_{0}\right)+Y
$$

Substituting equation (17) into (1) and applying boundary conditions (3), we have:

$$
\mathrm{U}(\mathrm{Y})=\frac{G}{6}\left(\frac{1}{4}-Y^{2}\right)\left[Y+3\left(\frac{1}{2}-\theta_{0}\right)\right]
$$

Equations (17) and (18) above give the conduction regime solution which is exactly the same as presented by Weidman and Magyari [5, 6] respectively.

\section{Results and Discussion}

In order to verify the accuracy of the present method, we obtained numerical values of equations (14) and (15), compare it with the solutions of Magyari [6] and then use the well-known implicit finite difference method (IFDM) to justify the results. This study has been performed over the reasonable range of $0 \leq \theta_{0} \leq 1 / 2$. The selected reference values of $\theta_{0}=0$ corresponds to the open slot while $\theta_{0}=1 / 2$ to capped slot, the aspect ratio $B=20$ at $G=1.0 \times 10^{6}$ and $C=0.5$ to determine $\tau$ from equation (4) for the present analysis as given in [5,6]. Tables 1 and 2 compare velocity and temperature profiles of the present work with those of Magyari [6] and IFDM for atmospheric air $(\operatorname{Pr}=0.72)$ and water $(\operatorname{Pr}=8.1)$ respectively. It is observed that the numerical values of both methods are exactly the same for both velocity and temperature profiles while the IFDM validates the results. For $(y>0)$, the velocity is seen to decrease with increase in $\theta_{0}$ but the reverse result is noticed for $(y \leq 0)$. The reversed result is observed for temperature profiles of atmospheric air $(\operatorname{Pr}=0.72)$. On the other hand, for water $(\operatorname{Pr}=8.1)$, velocity as well as temperature is seen to increase with increase in $\theta_{0}$ for $(y \neq 0)$.

where 
Table 1. Numerical comparisons of velocity profiles and temperature distributions of Magyari [6] and present work (D'Alembert) for atmospheric air (Pr $=$ $0.72)$.

\begin{tabular}{|c|c|c|c|c|c|c|c|}
\hline \multirow[b]{2}{*}{$\boldsymbol{\theta}_{\mathbf{0}}$} & \multirow[b]{2}{*}{$\boldsymbol{Y}$} & \multicolumn{3}{|c|}{ Velocity profiles } & \multicolumn{3}{|c|}{ Temperature distributions } \\
\hline & & $U(Y)[6]$ & $U(Y)$ present work & $U(Y)$ IFDM & $\theta(Y)[6]$ & $\theta(Y)$ present work & $\theta(Y)$ IFDM \\
\hline \multirow{3}{*}{0} & -0.2 & -12.44124 & -12.44124 & -12.5478 & 0.002767 & 0.002767 & 0.002711 \\
\hline & 0 & -101.2117 & -101.2117 & -99.6343 & -0.009642 & -0.009642 & -0.009678 \\
\hline & 0.2 & 403.7874 & 403.7874 & 404.3022 & -0.066382 & -0.066382 & -0.065722 \\
\hline \multirow{3}{*}{0.1} & -0.2 & -51.5759 & -51.5759 & -51.7233 & 0.009129 & 0.009129 & 0.009012 \\
\hline & 0 & -80.9694 & -80.9694 & -79.7074 & -0.007714 & -0.007714 & -0.007743 \\
\hline & 0.2 & 364.6528 & 364.6528 & 365.1269 & -0.066382 & -0.066382 & -0.059421 \\
\hline \multirow{3}{*}{0.2} & -0.2 & -90.7105 & -90.7105 & -90.8988 & 0.015490 & 0.015490 & 0.0153 \\
\hline & 0 & -60.7270 & -60.7270 & -59.7807 & -0.005785 & -0.005785 & -0.005807 \\
\hline & 0.2 & 325.5181 & 325.5181 & 325.9514 & -0.053659 & -0.053659 & -0.05312 \\
\hline \multirow{3}{*}{0.3} & -0.2 & -129.8451 & -129.8451 & -130.0742 & 0.021852 & 0.021852 & 0.02161 \\
\hline & 0 & -40.4847 & -40.4847 & -39.8538 & -0.003857 & -0.003857 & -0.003871 \\
\hline & 0.2 & 286.3835 & 286.3835 & 286.7760 & -0.053659 & -0.053659 & -0.04682 \\
\hline \multirow{3}{*}{0.4} & -0.2 & -168.9797 & -168.9797 & -169.2497 & 0.028213 & 0.028213 & 0.027915 \\
\hline & 0 & -20.2423 & -20.2423 & -19.9268 & -0.001928 & -0.001928 & -0.001936 \\
\hline & 0.2 & 247.2489 & 247.2489 & 247.6006 & -0.040936 & -0.040936 & -0.040518 \\
\hline \multirow{3}{*}{0.5} & -0.2 & -208.1143 & -208.1143 & -208.4253 & 0.034575 & 0.034575 & 0.034216 \\
\hline & 0 & 0 & 0 & 0 & 0 & 0 & 0 \\
\hline & 0.2 & 208.1143 & 208.1143 & 208.4250 & -0.034575 & -0.034575 & -0.034217 \\
\hline
\end{tabular}

Table 2. Numerical comparisons of velocity profiles and temperature distributions of Magyari [6] and present work (D'Alembert) for water(Pr = 8.1).

\begin{tabular}{|c|c|c|c|c|c|c|c|}
\hline \multirow[b]{2}{*}{$\boldsymbol{\theta}_{0}$} & \multirow[b]{2}{*}{$Y$} & \multicolumn{3}{|c|}{ Velocity profiles } & \multicolumn{3}{|c|}{ Temperature distributions } \\
\hline & & $U(Y)[6]$ & $U(Y)$ present work & $U(Y)$ IFDM & $\theta(Y)[6]$ & $\theta(Y)$ present work & $\theta(Y)$ IFDM \\
\hline \multirow[b]{2}{*}{0} & -0.2 & -0.0538 & -0.0538 & -0.0475 & -0.000013 & -0.000013 & -0.000014 \\
\hline & 0 & 1.1529 & 1.1529 & 1.0662 & 0.000192 & 0.000192 & 0.000209 \\
\hline \multirow{3}{*}{0.1} & -0.2 & 2.3647 & 2.3647 & 2.2733 & 0.000222 & 0.000222 & 0.000250 \\
\hline & 0 & 0.9223 & 0.9223 & 0.8530 & 0.000153 & 0.000153 & 0.000167 \\
\hline & 0.2 & -21.7134 & -21.7134 & -20.8396 & -0.002106 & -0.002106 & -0.002362 \\
\hline \multirow{3}{*}{0.2} & -0.2 & 4.7833 & 4.7833 & 4.5941 & 0.000458 & 0.000458 & 0.000514 \\
\hline & 0 & 0.6917 & 0.6917 & 0.6297 & 0.000115 & 0.000115 & 0.000126 \\
\hline & 0.2 & -19.2948 & -19.2948 & -18.5188 & -0.001871 & -0.001871 & -0.002098 \\
\hline \multirow{3}{*}{0.3} & -0.2 & 7.2019 & 7.2019 & 6.9149 & 0.000693 & 0.000693 & 0.000778 \\
\hline & 0 & 0.4612 & 0.4612 & 0.4265 & 0.000077 & 0.000077 & 0.000084 \\
\hline & 0.2 & -16.8762 & -16.8762 & -16.1980 & -0.001635 & -0.001635 & -0.001834 \\
\hline \multirow{3}{*}{0.4} & -0.2 & 9.6205 & 9.6205 & 9.2357 & 0.000929 & 0.000929 & 0.001042 \\
\hline & 0 & 0.2306 & 0.2306 & 0.2132 & 0.000038 & 0.000038 & 0.000042 \\
\hline & 0.2 & -14.4576 & -14.4576 & -13.8772 & -0.001399 & -0.001399 & -0.001570 \\
\hline \multirow{2}{*}{0.5} & -0.2 & 12.0391 & 12.0391 & 11.5565 & 0.001164 & 0.001164 & 0.001306 \\
\hline & 0.2 & -12.0391 & -12.0391 & -11.5565 & -0.001164 & -0.001164 & -0.001306 \\
\hline
\end{tabular}

Figure 1 presents temperature distributions at different values of reference temperature $\left(\theta_{0}\right)$ for fixed value of $\operatorname{Pr}=0.72$. It is observed that temperature decrease along both walls with increase in variation of reference temperature. Also, it is evident that the temperature of the open slot is seen to be higher than capped slot. Similar result is also noticed in Figure 3 when Prandtl number is fixed at $\operatorname{Pr}=8.1$. In addition, the center of the channel in Figures 3 and 4 for temperature and velocity of water respectively is seen to be independent on the value of reference temperature $\left(\theta_{0}\right)$.

Figures 2 and 4 depict velocity profiles at different values of reference temperature for fixed values of $\operatorname{Pr}=0.72$ and 8.1 respectively. It is found that there exist reverse flow at the hot wall and the maximum velocity is attained when $\theta_{0}=0$ which corresponds to open slot. This is true physically since the barrier to the flow is removed. In addition, this reverse flow is seen to be dependent on reference temperature $\left(\theta_{0}\right)$. Finally, two points of inflexions are observed in each Figures $1-4$, at these points, the temperature distributions as well as the velocity profiles is independent on the reference temperature $\left(\theta_{0}\right)$.

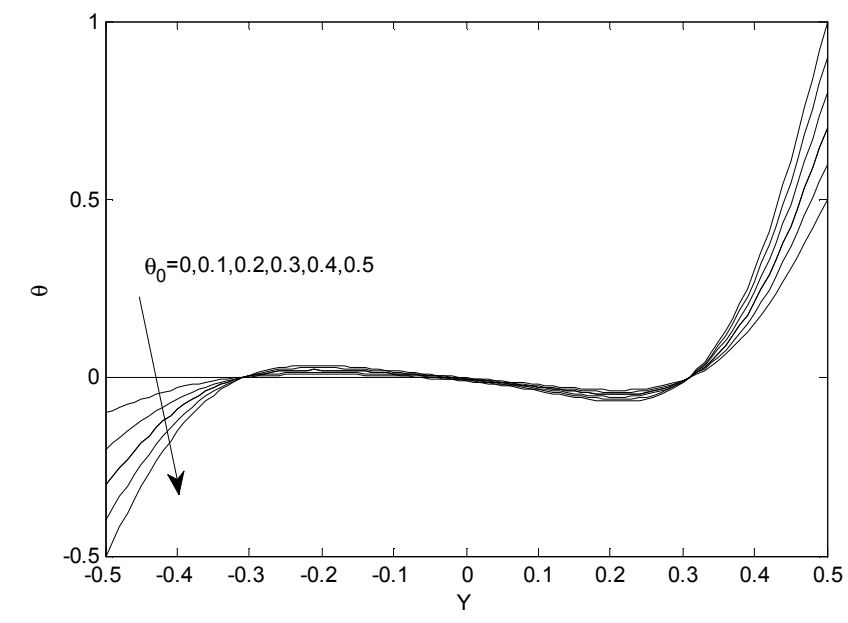

Figure 1. Temperature distribution versus $Y$ at different values of $\theta_{0}$ for $\operatorname{Pr}=0.72$. 


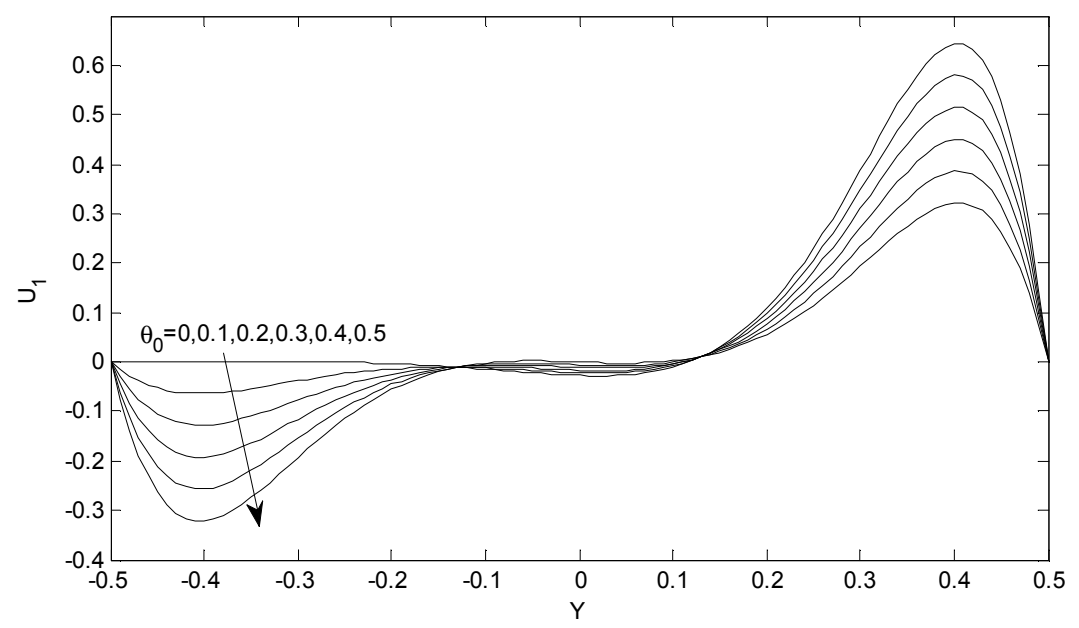

Figure 2. Velocity profiles versus $Y$ at different values of $\theta_{0}$ for $P r=0.72$.

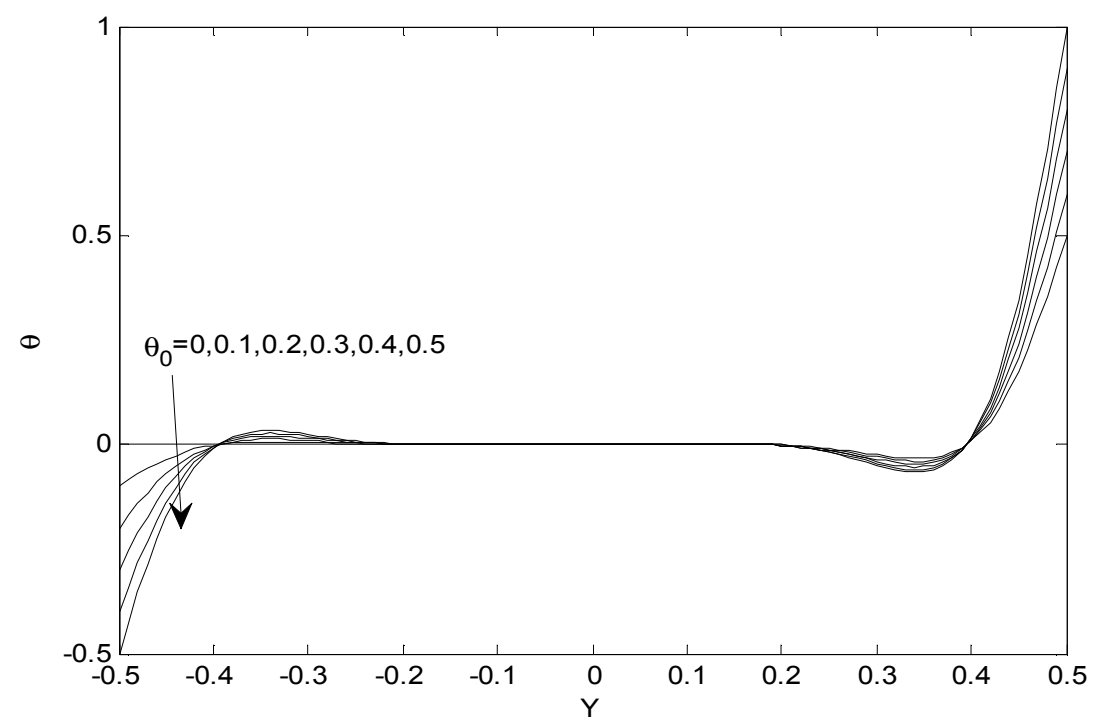

Figure 3. Temperature distributions versus $Y$ at different values of $\theta_{0}$ for $\operatorname{Pr}=8.1$.

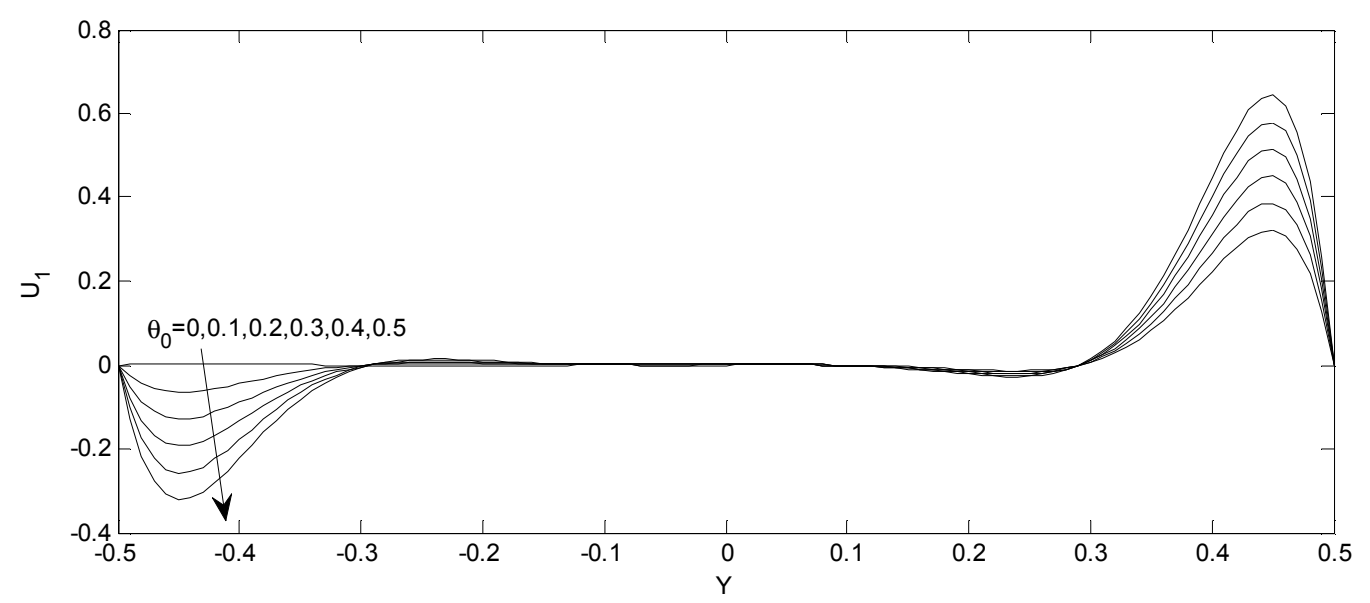

Figure 4. Velocity profiles versus $Y$ at different values of $\theta_{0}$ for $\operatorname{Pr}=8.1$.

\section{Conclusions}

Fully developed free convection flow in a vertical slot with open to capped ends discussed by Weidman and Magyari [5, 6] has been studied in detail in this paper. Closed-form solution for velocity and temperature profiles is obtained 
with the use of D'Alembert approach. Based on the numerical values obtained from the exact solutions, we draw the following conclusions:

1. D'Alembert method is a reliable, simple and accurate method of solution. This is based on the exactness of the numerical comparison in table 1 and 2 with Magyari [6]. The graphs plotted are also seen to be exact with figures 2 and 3 of Weidman [5].

2. As an accuracy check on the proposed method of solution, the conductive regime solution of equation (17) and (18) is seen to be exactly the same with Weidman [5] and Magyari [6]. Also, the numerical value of implicit finite difference method (IFDM) result is seen to be close to the exact solution.

\section{List of Symbols}

$\begin{array}{ll}B & \text { aspect ratio } \\ d & \text { gap width } \\ E & \text { Elder number } \\ \mathrm{g} & \text { gravitational constant } \\ l & \text { cavity height } \\ p & \text { fluid pressure } \\ \mathrm{Pr} & \text { Prandtl number } \\ U & \text { dimensionless velocity } \\ Y & \text { dimensionless coordinate }\end{array}$

\section{Greek Symbols}

$\beta \quad$ vertical temperature gradient

$\gamma \quad$ thermal expansion coefficient

$\Delta T \quad$ temperature contrast

$\theta \quad$ dimensionless temperature

$\theta_{0} \quad$ continuous variation of the reference temperature

$\kappa \quad$ thermal diffusivity

$\mu \quad$ absolute viscosity

$v \quad$ kinematic viscosity

$\rho \quad$ fluid density

$\tau \quad$ dimensionless vertical temperature gradient

\section{References}

[1] Batchelor G. K. (1964). Heat transfer by free convection across a closed cavity between vertical boundaries at different temperature. Quart Appl Math. 12:209-233

[2] Elder J. W. (1965). Laminar free convection in a vertical slot. J Fluid Mech 23:77-98

[3] Daniels P. G. (1987). Convection in a vertical slot. J Fluid Mech 176:419-441

[4] Bühler K. (2003). Special solutions of Boussinesq-equations for free convection flows in a vertical gap. Heat Mass Transfer 39:631-638

[5] Weidman P. D. (2006). Convection regime flow in a vertical slot: continuum solutions from capped to open ends. Heat Mass Transfer 43:103-109

[6] Magyari E. (2006). Normal mode analysis of the fully developed free convection flow in a vertical slot with open to capped ends. Heat Mass Transfer 43:827-832

[7] Jha, B. K., Oni, M. O., and Aina, B.: Steady fully developed mixed convection flow in a vertical micro-concentric-annulus with heat generating/absorbing fluid: an exact solution. Ain Shams Engineering Journal (2016) DOI: 10.1016/j.asej.2016.08.005

[8] Jha, B. K., M. O. Oni, Natural convection flow in a vertical tube inspired by time-periodic heating, Alexandria Eng. J. (2016), http://dx.doi.org/10.1016/j.aej.2016.08.025

[9] M. O. Oni, Combined effect of heat source, porosity and thermal radiation on mixed convection flow in a vertical annulus: An exact solution, Eng. Sci. Tech., Int. J. (2017), 20 (2017) 518-527, http://dx.doi.org/10.1016/j.jestch.2016.12.009

[10] B. K. Jha, C. A. Apere, (2013). Unsteady MHD two-phase Couette flow of fluid-particle suspension. Applied Mathematical Modelling 37:1920-1931

[11] Jha, B. K., M. O. Oni, Laminar Convection in a Uniformly Heated Vertical Porous Channel Revisited, Advances in Applied Sciences 2017; 2(3): 28-32 